\title{
A new application of the modal-Hamiltonian interpretation of quantum mechanics: the problem of optical isomerism
}

\author{
Sebastian Fortin - Olimpia Lombardi - Juan Camilo Martínez González* \\ CONICET - Universidad de Buenos Aires
}

\section{1.- Introduction}

The modal interpretations of quantum mechanics found their roots in the works of van Fraassen $(1972,1974)$, who claimed that the quantum state always evolves unitarily (with no collapse) and determines what may be the case: which physical properties the system may possess, and which properties the system may have at later times. On this basis, in the 1980s several authors presented realist interpretations that can be viewed as belonging to a "modal family": realist, non-collapse interpretations of the standard formalism of the theory, according to which any quantum system possesses definite properties at all times, and the quantum state assigns probabilities to the possible properties of the system. Given the contextuality of quantum mechanics (Kochen and Specker 1967), the members of the family differ to each other in its rule of definite-value ascription, which picks out, from the set of all observables of a quantum system, the subset of definite-valued properties, that is, the preferred context (see Lombardi and Dieks 2014 and references therein).

The traditional modal interpretations, based on the biorthogonal decomposition or on the spectral-decomposition of the quantum state (Kochen 1985, Dieks 1988, 1989, Vermaas and Dieks 1995) faced some difficulties. On the one hand, given the multiple factorizability of a given Hilbert space, their rules of definite-value ascription may lead to contradictions of the Kochen-Specker variety (Bacciagaluppi 1995, Vermaas 1997). This difficulty motivated the development of an atomic modal version, which postulates the existence of certain elemental building blocks that compose all quantum systems in nature (Bacciagaluppi and Dickson 1999), and of a perspectival version, according to which the properties of a physical system have a relational character and are defined with respect to another physical system that serves as a "reference system" (Bene and Dieks 2002). On the other hand, those traditional modal interpretations do not pick out the right properties for the apparatus in non-ideal measurements (Albert and Loewer 1990, 1991, 1993, Elby 1993, Ruetsche 1995); this was considered as a "silver bullet" for killing the modal interpretations (Harvey Brown, cited in Bacciagaluppi and Hemmo 1996). Perhaps these problems explain the decline of interest in

\footnotetext{
${ }^{*}$ This work is fully collaborative: the alphabetical order of the names does not mean priority.
} 
modal interpretations since the end of the 90's. Jeffrey Bub's preference for Bohmian mechanics in those days can be understood in this context: given the difficulties of the modal interpretations whose preferred context depends on the state of the system, the natural alternative for a realist is Bohmian mechanics, which can be conceived as a member of the modal family whose preferred context is a priori defined by the position observable (Bub 1997).

But position is not the only observable that can be appealed to in order to define the state-independent preferred context of a modal interpretation. The modal-Hamiltonian interpretation (MHI) (Lombardi and Castagnino 2008, Castagnino and Lombardi 2008) endows the Hamiltonian of a system with the role of selecting the subset of the definitevalued observables of the system. This makes the MHI immune to the non-ideal measurement's "silver bullet", since it not only accounts for ideal and non-ideal measurements, but it also supplies a criterion to distinguish between reliable and non-reliable measurements in the non-ideal case (Lombardi and Castagnino 2008, Ardenghi, Lombardi and Narvaja 2013, Lombardi, Fortin and López 2015). Moreover, the MHI rule of definitevalue ascription has been reformulated in an explicitly invariant form, in terms of the Casimir operators of the Galilean group (Ardenghi, Castagnino and Lombardi 2009, Lombardi, Castagnino and Ardenghi 2010), and the compatibility of the MHI with the theory of decoherence has been proved (Lombardi 2010, Lombardi, Fortin, Castagnino and Ardenghi 2012). From the ontological viewpoint, the MHI proposes an ontology without individuals, according to which quantum systems are bundles of properties, and properties inhabit the realm of possibility, not less real than the domain of actuality (da Costa, Lombardi and Lastiri 2013, da Costa and Lombardi 2014, Lombardi and Dieks 2016).

Nevertheless, in spite of the many aspects covered by the MHI, perhaps its main advantage in the eyes of scientists is given by its several applications to well-known physical situations, leading to results compatible with experimental evidence: free particle with spin, harmonic oscillator, hydrogen atom, Zeeman effect, fine structure, Born-Oppenheimer approximation (see Lombardi and Castagnino 2008, Section 5). The purpose of this paper is to add a new application to the list: the case of optical isomerism, which is a central issue for the philosophy of physics and of chemistry since it points to the core of the problem of the relationship between physics and chemistry. Here it will be shown that the MHI supplies a direct and physically natural solution to the problem, which does not require putting classical assumptions in "by hand." 
With this purpose, the paper is organized as follows. In Section 2, the discussion about whether chemistry is reducible to physics will be introduced in terms of the problem of molecular structure. On this basis, Section 3 will focus on the particular problem of optical isomerism and the so-called Hund's paradox, which points to the difficulty in giving a quantum explanation to chirality. Section 4 will be devoted to explain the different attempts to solve the paradox and their difficulties. In Section 5, the main features of the MHI will be recalled, emphasizing the aspects that will lead, in Section 6, to offer a solution of Hund's paradox in exclusively quantum terms. Finally, in the Conclusions the general argument will be reviewed, stressing why the MHI supplies a perspective that sounds natural to chemists' ears and provides them the tools to face some general problems related with the links between chemistry and physics.

\section{2.- Linking physics and chemistry: the problem of molecular structure}

Since the advent of quantum mechanics and its application to chemical systems, reduction became a regulative idea in the accounts of the relationship between physics and chemistry. In the famous introductory paragraph of his article of 1929, Paul Dirac described the central idea of what later would be known as quantum chemistry: "The underlying physical laws necessary for the mathematical theory of a large part of physics and the whole of chemistry are thus completely known, and the difficulty is only that the exact application of these equations leads to equations much too complicated to be soluble. It therefore becomes desirable that approximate practical methods of applying quantum mechanics should be developed, which can lead to an explanation of the main features of complex atomic systems without too much computation" (Dirac 1929, p. 714). The approximate methods referred to by Dirac, which are the core of quantum chemistry, constitute the field in which the problem of the relationship between physics and chemistry acquires particular relevance.

The problem finds one of its main manifestations in the debate about the nature of molecular structure, given by the spatial arrangement of the nuclei in a molecule. The debate focuses not on an auxiliary or secondary notion, but on a central concept of molecular chemistry: molecular structure is the main factor in the explanation of reactivity, it is "the central dogma of molecular science” (Woolley 1978, p. 1074). As Robin Hendry claims, "molecular structure is so central to chemical explanation that to explain molecular structure is pretty much to explain the whole of chemistry" (Hendry 2010, p. 183). The problem consists in the fact that the concept seems to find no place in the theoretical framework of quantum mechanics. Whereas " $[\mathrm{t}]$ he alpha and omega of molecular chemistry is the doctrine 
that molecules exist as individual objects and that every molecule has a shape, characterized by its molecular frame" (Primas 1994, p. 216), the classical idea of definite spatial position for the atomic nuclei, conceived as individual objects, is, at least, strongly conflictive in the quantum context. Although this problem has been widely discussed, the debate is far from settled.

Some authors consider that the impossibility of deriving molecular structure from quantum mechanics is the consequence of our partial knowledge of the molecular systems in the theoretical framework of quantum mechanics. For instance, Guy Woolley and Brian Sutcliffe stress that: "We have never claimed that molecular structure cannot be reconciled with or reduced to quantum mechanics, or that there is something 'alien' about it; our claim is much more modest. We do not know how to make the connection." (Sutcliffe and Woolley 2011, p. 94; see also Sutcliffe and Woolley 2012). Hinne Hettema (2012) is even more optimistic: he adopts an explicitly reductionist stance by considering that the intertheoretic relationship between molecular chemistry and quantum mechanics fulfill the conditions required by the traditional Nagelian model of reduction. Another reductionist strategy is that based on the concept of quantum decoherence: conceived as the process that accounts for the classical limit of quantum mechanics (Zurek 1991, 2003), environment induced decoherence would supply the necessary link between the classical concepts of molecular chemistry and the quantum domain (Trost and Hornberger 2009, Scerri 2011, 2013).

From an opposite position, other authors point out the difficulties of explaining molecular structure in exclusively quantum terms. This is the case of Woolley in his works of the 70's and the 80 's, where he stresses that, by means of the description of a molecule from "first principles", "one cannot even calculate the most important parameters in chemistry, namely, those that describe the molecular structure" (Woolley 1978, p. 1074); he considers that the impossibility of determining the geometry of a molecule by means of quantum mechanics is a proof of the fact that molecular structure is only a "powerful and illuminating metaphor" (Woolley 1982, p. 4). In turn, Hans Primas highlights non-locality as a specific feature of quantum mechanics that excludes the spatial concept of molecular structure: "the holistic correlations between the nuclei and electrons are suppressed, so the description of a molecule reduces to the description of the motion in the electrical field of a classical nuclear framework" (Primas 1998, p. 91; see also Primas 1983). Following Primas' ideas, Robert Bishop (2005) also recognizes the limitations of quantum mechanics to account for molecular structure, and points out that proper attention to the context relevant to a particular situation can resolve otherwise intractable problems (see also Bishop and Atmanspacher 2006). Other 
authors stress that conceiving the molecule as an individual object with its own spatial structure requires to ignore quantum correlations: "The shape of a molecular state should of course not show holistic correlations to other molecular quantities and hence be unambiguously defined." (Amann 1992, p. 32).

Robin Hendry (2004, 2008, 2010) has largely addressed the issue of molecular structure in the context of the problem of reduction. He clearly distinguishes between the intertheoretic and the metaphysical aspects of the reduction debate; this distinction allows him to point out that, although reductionists and non-reductionists agree that classical intertheoretic reductions of chemistry are not currently available, they differ in how they interpret the situation: "the issue is essentially future directed -both sides must wait and see, even if they would bet different ways. But why do the two sides make different bets? Perhaps the answer concerns their different underlying metaphysical views." (Hendry 2010, p. 184). On this basis, Hendry considers that the reduction debate must turn to consider the ontological relationships between the entities, processes, and laws studied by different sciences.

A central element in the discussion about molecular structure is the role played by the Born-Oppenheimer approximation, whose fundamental premise is the possibility of decomposing the Hamiltonian of the molecule into its electronic and its nuclear components. This move relies on assuming the nuclei as classical-like particles at rest in a definite position: on this basis, the terms of kinetic energy associated to the nuclear motion are removed (clamped-nuclei assumption). In the Hamiltonian resulting from that assumption, the molecular structure is described by the positions of the nuclei. In turn, the so-called 'potential energy surface' that affects the electrons of the molecule can be calculated by means of this Hamiltonian. Nevertheless, from the viewpoint of reduction, the Born-Oppenheimer approximation faces at least two difficulties. First, it introduces the molecular structure into the quantum description from the very beginning, since the positions of the nuclei are established with the appeal to classical geometric considerations. Second, the assumption of the nuclei at rest in fixed spatial positions contradicts the Heisenberg principle, which prevents quantum systems from having definite values of position and velocity simultaneously (see Lombardi and Castagnino 2010, Chang 2015). As Hendry (1998, 2010) points out, the "proxy" defense of Born-Oppenheimer models is based on the assumption that using them instead of the exact solution makes only a small difference to the energy; but, from a theoretical viewpoint, those models "simply assume the facts about molecular structure that ought to be explained" (Hendry 2010, p. 186). Hasok Chang clearly makes the point: "The difficulty here is not only about the practicalities of the calculation, and the 
clamping-down of nuclei is not merely an approximation. Aside from assuming that the nuclei are fixed, it is necessary to know exactly where exactly the nuclei in question should be placed. Otherwise it is not possible to specify the potential function, which needs to be inserted into the Schrödinger equation, whose solution determines the wavefuncion of the electrons in the molecule. In other words, without knowing the locations of the nuclei in the molecule it is impossible even to set up (not to mention solve) the Schrödinger equation." (Chang 2015, p. 198).

The discussion around the nature of molecular structure is often related with the interpretation of the Born-Oppenheimer approximation. However, there is a specific problem regarding molecular structure that plays a central role in the debate about the relationship between molecular chemistry and quantum mechanics. This is the problem of isomerism. The particular relevance of this case is that, as it will be explained in the next section, the difficulties derived from it are independent from the assumptions involved in the BornOppenheimer approximation.

\section{3.- Isomerism and optical activity: Hund's paradox}

The composition of a molecule is given by its chemical formula, which specifies in what stechiometric proportion the component elements are present in the chemical compound. But the formula supplies no information about the geometrical arrangement of the component atoms. For instance, $\mathrm{C}_{2} \mathrm{H}_{4} \mathrm{O}_{2}$ corresponds to different chemical compounds: methyl formate, acetic acid and glycolaldehyde. Compounds that contain the same number of atoms of each element (and, therefore, have the same chemical formula) but have different spatial arrangements of their atoms are called isomers (see Figure 1). Isomerism is a phenomenon highly relevant in chemistry, since it explains the difference in the physical and chemical behaviors of substances with the same composition.

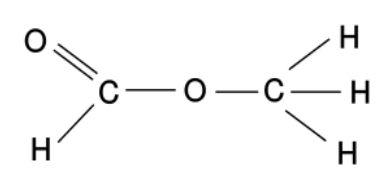

Methyl formate<smiles>CC(=O)O</smiles>

Acetic acid<smiles>O=CCO</smiles>

Glycolaldehyde

Figure 1: Isomers corresponding to the chemical formula $\mathrm{C}_{2} \mathrm{H}_{4} \mathrm{O}_{2}$

There are two main forms of isomerism: structural isomerism and stereoisomerism. In 
structural isomers, sometimes referred to as constitutional isomers, the component atoms and the functional groups are connected together in different ways. On the other hand, in stereoisomers, the component atoms are linked together in the same way, but the geometrical positioning of atoms and functional groups in space differs. The class of stereoisomers includes the subclass of enantiomers: the structures of the members of a pair of enantiomers are non-superimposable mirror-images of each other (see Figure 2 below). The property that distinguishes the members of a pair of enantiomers is called chirality.

When present in a symmetric environment, enantiomers have identical chemical and physical properties. Nevertheless, they differ in the kind of interaction with polarized light. In particular, the members of a pair of enantiomers can be distinguished for their ability to rotate plane-polarized light by equal amounts but in opposite directions. It is for this reason that they are called optical isomers and it is usually said that they are optically active. When the plane of polarization is rotated clockwise (as seen by a viewer towards whom the light is traveling), the optical isomer is called dextro-rotatory (D); in the case of counterclockwise rotation, the optical isomer is called levo-rotatory $(\mathrm{L})$.

Chiral molecules have an important function in the enzymatic reactions of biological systems: many pharmacological drugs are chiral, and generally only one of the members of the pair exhibits biological activity. The action of drugs is usually explained by means of the idea of "receptor". Receptors are protein molecules in human body, which are exclusively built from L-aminoacids. This means that protein molecules are chiral and, as a consequence, they have different reactions with the two enantiomers of a chiral drug. For instance, aspartame is a sweetening agent that has two enantiomers: one of them is more than a hundred times sweeter than sucrose; the other, is tasteless or slightly bitter. A dramatic example of the different biological activity of enantiomers is that of thalidomide, a drug of molecular formula $\mathrm{C}_{13} \mathrm{H}_{10} \mathrm{~N}_{2} \mathrm{O}_{4}$ introduced to the marked in 1957 by a pharmaceutical company in West Germany. It was prescribed to pregnant women as a sedative and to prevent nausea; its use spread rapidly to 46 countries. But the drug was made and marketed as a mixture of Dthalidomide and L-thalidomide: the D-thalidomide is an effective sedative, whereas the Lthalidomide is a teratogen, that is, an agent affecting the development of the fetus and causing structural or functional abnormality. It is not known exactly how many worldwide victims of the drug there have been, although estimates range from 10.000 to 100.000 . These are only some examples of the fact that, at present, the control and production of enantiomers is a key activity in the pharmaceutical industry.

Chemists not only successfully explain the behavior of enantiomers in terms of the 
geometrical shape of the molecules, but they have also developed many techniques for the preparation of compounds where the proportions of the enantiomers can be controlled. The problem with chirality arises not at the chemical level, but with the attempts to explain the phenomenon in quantum terms.

When a molecule is described by quantum mechanics, the Coulombic Hamiltonian only depends on the distances between the particles composing the molecule; in particular, if only the atomic nuclei are considered, the Hamiltonian depends exclusively on the inter-nuclear distances. In the case of structural isomers, the difference between them is manifest in the Hamiltonian. But in the case of optical isomerism, all the inter-atomic distances are the same for the two members of the pair and, as a consequence, the Hamiltonian is exactly the same for both. This means that quantum mechanics supplies the same description for two structures that can effectively be distinguished in practice through their optical and biological activity.

It is very important to stress that the problem of optical isomerism is completely independent of the Born-Oppenheimer approximation. Let us suppose, for instance, that we could count with the exact Hamiltonian $H$ (with no approximation) of a molecule of alanine $\mathrm{C}_{3} \mathrm{H}_{7} \mathrm{NO}_{2}$, which includes three nuclei of carbon, one of nitrogen, and two of oxygen, plus all its electrons (Figure 2)
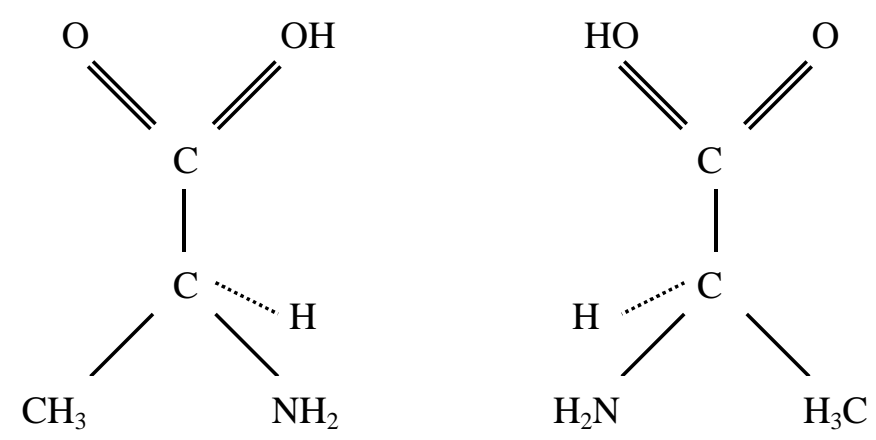

Figure 2: Enantiomers of alanine

Even if we cannot write down this Hamiltonian due to its complexity, we know that it only depends on the distance of the component particles and, therefore, not even the exact Hamiltonian can account for the difference between D-alanine and L-alanine. As Sutcliffe and Woolley claim: "Clearly then, an eigenstate of $H$ does not correspond to a classical molecule with structure! That observation begs the question: what are the equations that determine the quantum state of molecules? Beyond the BO [Born-Oppenheimer] approximation we have no idea." (Sutcliffe and Woolley 2012, p. 416; emphasis in the original). In short, the problem of 
the quantum mechanical distinction of optical isomers of the same substance is beyond the Born-Oppenheimer approximation and its underlying assumptions.

The problem embodied in optical isomerism points to a deep difficulty in the attempts to account for molecular structure in quantum terms. This was already noticed by Friedrich Hund, pioneer in the development of quantum chemistry, in the 1920s. Hund's paradox can be formulated in two versions. The first one, due to Hund himself (1927), reads as follows: since chiral states are not eigenstates of the Hamiltonian (the Hamiltonian is parity invariant), and none of them corresponds to the basal state, why do certain chiral molecules display an optical activity that is stable in time, associated to a well-defined chiral state, and are they not in a superposition of the two possible chiral states? More recently, Hund's paradox was formulated in a slightly stronger version (Berlin, Burin and Goldanskii 1996): why do chiral molecules have a definite chirality?

Let us consider a chiral molecule from the perspective of quantum mechanics, and call $H$ its total Hamiltonian, which takes into account all the interactions among nuclei, among electrons and among electrons and nuclei. Since the Coulombic interaction only depends on the distance between the interacting particles, it is symmetric under spatial reflection; therefore, the Hamiltonian commutes with the parity operator $P$ :

$$
[P, H]=0
$$

This means that the eigenstates of the Hamiltonian have definite parity. Moreover, this feature is preserved during the time evolution of the system, because the parity operator commutes with the Hamiltonian and, as a consequence, is a constant of motion.

With these elements, Hund's paradox can be expressed in formal terms. The eigenstates $\left|\omega_{n}\right\rangle$ of the Hamiltonian of this molecule have parity symmetry:

$$
P\left|\omega_{n}\right\rangle= \pm\left|\omega_{n}\right\rangle
$$

In particular, the even levels have even parity, and the odd levels have odd parity. For instance, the ground state $\left|\omega_{0}\right\rangle$ is symmetric and the first excited state $\left|\omega_{1}\right\rangle$ is anti-symmetric:

$$
P\left|\omega_{0}\right\rangle=+\left|\omega_{0}\right\rangle \quad P\left|\omega_{1}\right\rangle=-\left|\omega_{1}\right\rangle
$$

However, on the basis of experimental data it is possible to know that the states of optical isomers do not have this symmetry. In fact, if the two isomers corresponding to the two chiral states are represented by the quantum states $|L\rangle$ and $|D\rangle$, each isomer is the mirror image of the other, that is: 


$$
\begin{aligned}
& P|L\rangle=|D\rangle \\
& P|D\rangle=|L\rangle
\end{aligned}
$$

Therefore, the states $|L\rangle$ and $|R\rangle$ cannot be eigenstates of the Hamiltonian. On the contrary, they can be expressed as superposition of $\left|\omega_{0}\right\rangle$ and $\left|\omega_{1}\right\rangle$ :

$$
\begin{aligned}
& |L\rangle=\frac{1}{\sqrt{2}}\left(\left|\omega_{0}\right\rangle+\left|\omega_{1}\right\rangle\right) \\
& |D\rangle=\frac{1}{\sqrt{2}}\left(\left|\omega_{0}\right\rangle-\left|\omega_{1}\right\rangle\right)
\end{aligned}
$$

On the other hand, the ground state is a superposition of the chiral states:

$$
\left|\omega_{0}\right\rangle=\frac{1}{\sqrt{2}}(|L\rangle+|R\rangle)
$$

Why, then, do we observe chiral states? In other words: "What is the shape of the hypothetical superposition of these two species? Is there a proper reason to exclude such superpositions? What reason can be given from a quantum-mechanical point of view-for chemical systematics?" (Amann 1992, p. 32).

\section{4.- Some attempts to solve the paradox}

Once it is concluded that chiral states are not eigenstates of the Hamiltonian, it is necessary to explain why those states are observed in the laboratory. One strategy consists in maintaining the Coulombic Hamiltonian, identifying the states $|L\rangle$ and $|D\rangle$ as superpositions of the eigenstates of the Hamiltonian, and then supplying a reason why the molecule does not decay to the ground state, eigenstate of the Hamiltonian: this is Hund's strategy, based on graphical visualization.

Let us consider a quantum system with a potential $V(x)$ with mirror reflection symmetry, such as that of Figure 3. The states "at rest on the left" and "at rest on the right" break the symmetry of the situation and, for this reason, they cannot be eigenstates of the Hamiltonian.

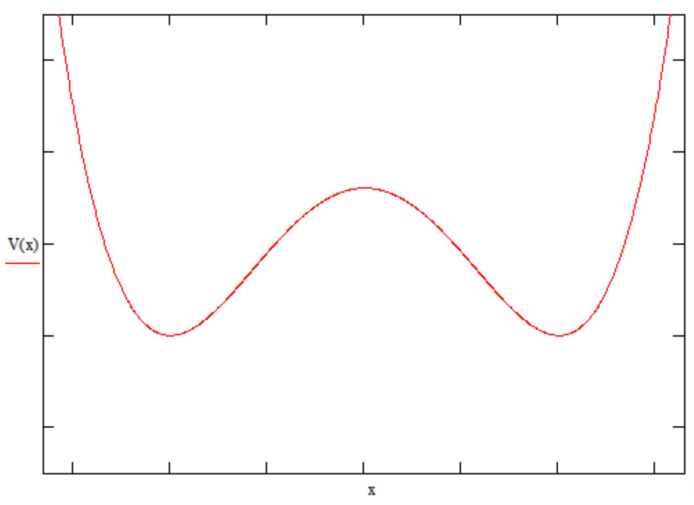

Figure 3: Potential $V(x)$ with mirror symmetry 
In fact, if the wavefunctions of the ground state $\left|\omega_{0}\right\rangle$ and of the first excited state $\left|\omega_{1}\right\rangle$ are graphed, the results of Figure 4 are obtained.
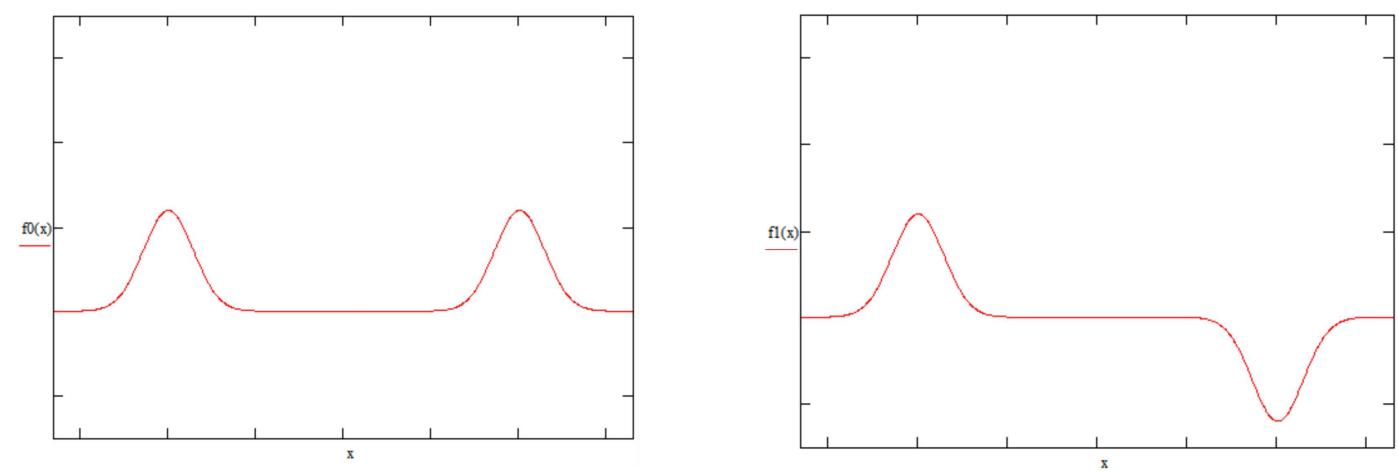

Figure 4. Shape of the eigenfuncions of a particle subject to the potential $V(x)$. On the left, the ground state, symmetric under space reflections. On the right, the first excited state, antisymmetric under space reflections.

Figure 4 clearly shows that the eigenfunctions of the Hamiltonian preserve parity: they do not correspond to states localized on the left or on the right, since both occupy both "wells." However, experimentally it is possible to place a quantum particle in one of the two minima of the potential, for instance, on the left. It is clear that this particle is not in an eigenstate of the Hamiltonian, but is in a superposition. The chiral states $|L\rangle$ and $|D\rangle$ correspond to wavefunctions as those shown in Figure 5.
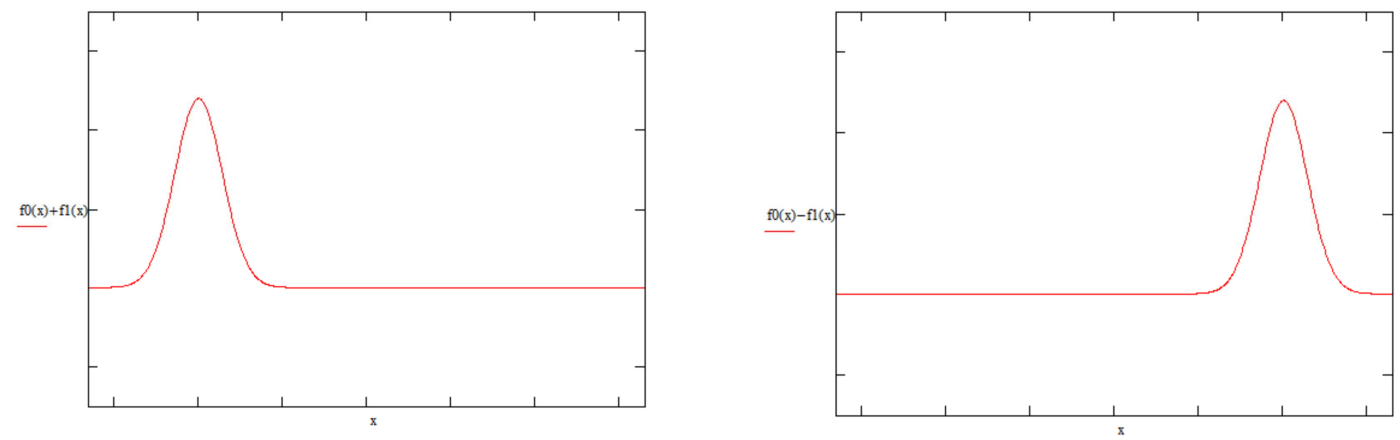

Figure 5. Shape of the eigenfuncions of a particle in the states $|L\rangle$ and $|R\rangle$. On the left, the state $|L\rangle$ corresponds to a particle located on the left side. On the right, the state $|R\rangle$ corresponds to a particle located in the right side.

In their article "On the time dependence of optical activity", Robert Harris and Leo Stodolsky (1981) face the problem of chiral isomers and emphasize the limitations of Hund's proposal. In particular, since chiral states are not eigenstates of the Hamiltonian, it is necessary to admit the existence of an exceptional kind of molecules that do not remain in their ground states. For these authors, the key to solve this difficulty is the interaction between 
molecules; they modify the Hamiltonian of the system with the purpose to describe such interaction. The central idea is that the paradox arises when the molecule is considered in isolation, whereas a real system actually consists of many molecules in interaction. For this reason, they propose to study the molecules in collision to each other: intermolecular collisions would modify the dynamics of the whole composite system.

The solution to Hund's paradox offered by Harris and Stodolsky is based on considering the interaction of the molecule with its environment, in this case composed of other similar molecules. But in this case it is not clear how the interaction breaks the symmetry of the problem. As Hendry claims: "The particular form of the symmetry-breaking addition must be justified however, and it is quite mysterious how that could work if all one has in the environment are more molecules described by Coulombic Hamiltonians. The Coulomb Schrödinger equation for an n-molecule ensemble of hydrogen chloride molecules has precisely the same symmetry properties as a Coulomb Schrödinger equation for a 1-molecule system. If the particular form of the symmetry-breaking addition is not justified, then it is just ad hoc: a deus ex machina." (Hendry 2010, p. 186).

Once the possibility of solving the problem of optical isomerism in terms of the interaction with an environment was considered, it did not take long for the idea of decoherence to enter the stage (see, e.g., Joos 1996). In fact, although the ground state of the molecule is a superposition of the chiral states $|L\rangle$ and $|D\rangle$, we always measure definite values of chirality. Analogously to the traditional quantum measurement problem, here the problem is to account for the transition from the superposition to one of the chiral states, say, $|L\rangle$ :

$$
\left|\omega_{0}\right\rangle=\frac{1}{\sqrt{2}}(|L\rangle+|D\rangle) \rightarrow|L\rangle
$$

During the last decades, the quantum measurement problem is being faced in the light of the theory of environment induced decoherence (Zurek 1981, 1991, 2003), which relies on the study of the effects of the interaction between a quantum system, considered as an open system, and its environment. On the basis of the analysis of the evolution of the reduced state of the open system, it is proved that, under certain conditions, that state becomes diagonal, that is, loses the interference terms that preclude classicality. The reduced state is conceived as a mixed state containing only the representation of classical correlations and, as a consequence, it can be interpreted in terms of ignorance. When this idea is applied to the problem of isomerism, the conclusion is that the molecule is in one of the states $|L\rangle$ or $|D\rangle$, and the probabilities measure our ignorance about its definite state. In this way, the theory of 
decoherence would have solved the problem underlying Hund's paradox.

This idea was widely accepted in the philosophy of chemistry community. For instance, in the Editorial 37 of the journal Foundations of Chemistry, its editor Eric Scerri (2011) explicitly considers optical isomerism in the context of the debate about the possibility of accounting molecular structure in quantum-mechanical terms. He relates the problem of isomerism with the measurement problem in quantum mechanics, and states that the question about molecular structure "is part of a bigger problem that has long plagued the foundations of quantum mechanics, namely the problem of the collapse of the wavefunction. [...] this problem has gradually begun to dissolve with the growing realization of the role of quantum decoherence in physics and other disciplines." (Scerri 2011, p. 4). On the basis of the extensive literature on decoherence, Scerri claims that the problem of optical isomerism is dissolved by taking into account the interaction of the molecule with its environment: "The study of decoherence has shown that it is not just observations that serve to collapse the superpositions in the quantum mechanics. The collapse can also be brought about by molecules interacting with their environment." (Scerri 2011, p. 4; for a similar claim, see Scerri 2013).

This position, however, does not take into account that, in spite of the wide application of the decoherence program, its ability to solve the traditional measurement problem has been largely discussed, and doubts have been raised on the basis of different arguments. In fact, no matter how the open molecule evolves, the state of the whole system molecule+environment is a superposition at any time: the superposition never disappears through its unitary evolution. For this reason, although the reduced state operator of the molecule lacks interference terms, this does not allow us to suppose that what is observed at the end of the process is one of two definite events: either the event associated with $|L\rangle$, or the event associated with $|D\rangle$. In this sense, Stephen Adler concludes: "I do not believe that either detailed theoretical calculations or recent experimental results show that decoherence has resolved the difficulties associated with quantum measurement theory" (Adler 2003, p. 136). The criticism of Jeffrey Bub (1997) is even stronger: stating that what is observed at the end of the measurement process is a definite event not only is unjustified, but also contradicts the eigenstate-eigenvalue link, a standard assumption in quantum mechanics.

Another way to criticize the solution to the measurement problem via decoherence relies on stressing the difference between a proper mixture -the mixed state of a closed system- and an improper mixture -the state of an open system, obtained by tracing off the degrees of freedom of its environment- (d'Espagnat 1966, 1976): improper mixtures cannot be 
interpreted in terms of ignorance. As Maximilian Schlosshauer emphasizes in his well-known book about decoherence: "Since the two systems $A$ and $B$ are entangled and the total composite system is still described by the superposition, it follows from the standard rules of quantum mechanics that no individual definite state can be attributed to either one of the subsystems. Reduced density matrices of entangled subsystems therefore represent improper mixtures" (Schlosshauer 2007, p. 48). The difference between proper and improper mixtures turns out to be even clearer when it is proved that the reduced state of the open system, obtained by partial trace, is a kind of coarse-grained state of the composite system to which the open system belongs (Fortin and Lombardi 2014). Perhaps confusions are due to the fact that proper and improper mixtures cannot be distinguished from a mathematical viewpoint. However, that mathematical indistinguishability essentially depends on the formalism used to express the theory, in particular, the Hilbert space formalism. But the situation might be different in other cases. For instance, it has been proved (Masillo, Scolarici and Sozzo 2009) that proper and improper mixtures are represented by different density operators in the socalled quaternionic formulation of quantum mechanics (Adler 1995); hence, in this formalism they can be distinguished also from a mathematical viewpoint. In a recent article, Scerri (2012) recognizes that the question of whether decoherence explains quantum measurement is a subtle matter, and refers to the review paper of Guido Bacciagaluppi (2012); in this paper the author points out that, although naive claims of the kind that decoherence gives a complete solution to the measurement problem are still somewhat part of the "folklore" of the matter, decoherence as such does not provide that solution, at least not unless it is combined with an appropriate interpretation of quantum mechanics (for a detailed argument, see Fortin, Lombardi and Martínez González 2016).

In summary, the different answers to the problem of optical isomerism offered in the literature are far from conclusive. Perhaps it is time to try with an interpretive strategy: tackling the problem by means of an interpretation of quantum mechanics capable to be applied not only to the abstract model of quantum measurement, but also to situations commonly treated in the practice of physics and chemistry.

\section{5.- The modal-Hamiltonian interpretation}

As advanced in the Introduction, the MHI is a realist, non-collapse interpretation belonging to the "modal family", which endows the Hamiltonian with a key role both in the definition of systems and subsystems and in the selection of the preferred context. 


\section{1- The postulates of the MHI}

By adopting an algebraic perspective, the MHI defines a quantum system $S$ as a pair $(\mathcal{O}, H)$ such that (i) $\mathcal{O}$ is a space of self-adjoint operators acting on a Hilbert space $\mathcal{H}$, representing the observables of the system, (ii) $H \in \mathcal{O}$ is the time-independent Hamiltonian of the system $S$, and (iii) if $\rho_{0} \in \mathcal{O}^{\prime}$ (where $\mathcal{O}^{\prime}$ is the dual space of $\mathcal{O}$ ) is the initial state of $S$, it evolves according to the Schrödinger equation.

A quantum system so defined can be decomposed in parts in many ways; however, not any decomposition will lead to parts which are, in turn, quantum systems. The expression 'tensor product structure' (TPS) is used to call any partition of a closed system $S$, represented in the Hilbert space $\mathcal{H}=\mathcal{H}_{A} \otimes \mathcal{H}_{B}$, into parts $S_{A}$ and $S_{B}$ represented in $\mathcal{H}_{A}$ and $\mathcal{H}_{B}$ respectively. Nathan Harshman and Sujeev Wickramasekara (2007a, 2007b) point out that quantum systems admit a variety of TPSs, each one leading to a different entanglement between their parts. However, there is a particular TPS that is invariant under time evolution: the TPS is dynamically invariant when there is no interaction between the parts. In other words, in the dynamically invariant case the components' behaviors are dynamically independent from each other; each one evolves unitarily according the Schrödinger equation. On this basis, according to the MHI, a quantum system can be split into subsystems when there is no interaction among the subsystems.

Composite systems postulate: A quantum system $S:(\mathcal{O}, H)$, with initial state $\rho_{0} \in \mathcal{O}^{\prime}$, is composite when it can be partitioned into two quantum systems $S^{1}:\left(\mathcal{O}^{1}, H^{1}\right)$ and $S^{2}:\left(\mathcal{O}^{2}, H^{2}\right)$ such that (i) $\mathcal{O}=\mathcal{O}^{1} \otimes \mathcal{O}^{2}$, and (ii) $H=H^{1} \otimes I^{2}+I^{1} \otimes H^{2}$, (where $I^{1}$ and $I^{2}$ are the identity operators in the corresponding tensor product spaces). In this case, we say that $S^{1}$ and $S^{2}$ are subsystems of the composite system $S=S^{1}+S^{2}$. If the system is not composite, it is elemental.

With respect to the preferred context, the basic idea of the modal-Hamiltonian interpretation is that the Hamiltonian of the system, with its own symmetries, defines the subset of observables that acquire definite actual values. The group of transformations that leave the Hamiltonian invariant is usually called "Schrödinger group" (Tinkham 1964). Since each symmetry of the Hamiltonian leads to an energy degeneracy, much valuable information on the energy spectrum of the system can be obtained by applying the machinery of the group theory to the study of the symmetries of the Hamiltonian. The degeneracies with origin in symmetries are called "normal" or "systematic" (Cohen-Tannoudji, Diu and Lalöe 1977). On 
the contrary, degeneracies that have no obvious origin in symmetries are called "accidental". However, deeper study usually shows either that the accidental degeneracy is not exact, or else that a hidden symmetry in the Hamiltonian can be found which explains the degeneracy. A classical example is the degeneracy, in the hydrogen atom, of states of different angular momentum $l$ but the same principal quantum number $n$ (for instance, $2 s$ and $2 p$ functions). In this case, Vladimir Fock (1935) showed that the degeneracy arises from a four-dimensional rotational symmetry of the Hamiltonian in momentum space. For this reason it is assumed that, once all the symmetries of the Hamiltonian have been considered, a basis for the Hilbert space of the system is obtained and the "good quantum numbers" are well defined. This strategy is what underlies the group approach to quantum mechanics, where the physical features of the quantum system are studied by analyzing the symmetry properties of its Hamiltonian (Weyl 1950, Tung 1985).

Now we have all the conceptual elements necessary to present the MHI rule of definitevalue ascription, here called 'actualization rule'. The basic idea can be expressed by the classical Latin maxim "Ubi lex non distinguit, nec nos distinguere debemus": where the law does not distinguish, neither ought we to distinguish. The Hamiltonian of the system, with its symmetries, is what rules actualization; then, none observable whose eigenvalues would distinguish among eigenvectors corresponding to a single degenerate eigenvalue of the Hamiltonian has to acquire definite value, since its actualization would introduce in the system an asymmetry not contained in the Hamiltonian. Once this idea is understood, the actualization rule can be formulated in a very simple way.

Actualization rule: Given an elemental quantum system $S:(\mathcal{O}, H)$, the actualvalued observables of $S$ are $H$ and all the observables commuting with $H$ and having, at least, the same symmetries as $H$.

The justification for selecting the Hamiltonian as the preferred observable ultimately lies in the physical relevance of the MHI and in its ability to solve interpretive difficulties. Here we will focus on the first point, in order to show that the case of Hund's paradox can be counted as a further successful application of the interpretation.

\section{2- Measuring as breaking symmetries}

As already mentioned, the MHI scheme has been applied to several well-known physical situations, leading to results consistent with empirical evidence (see Lombardi and Castagnino 
2008, Section 5). Let us recall some of them, in order to stress the role played by symmetries in those situations.

The Hamiltonian of the free particle reads

$$
H_{\text {free }}=\frac{P^{2}}{2 m}=\frac{P_{x}^{2}+P_{y}^{2}+P_{z}^{2}}{2 m}
$$

where $P$ is the momentum observable, with components $P_{x}, P_{y}, P_{z}$, and $m$ is the mass of the particle. $H_{\text {free }}$ is invariant under space-displacements in any direction, and the components $P_{x}, P_{y}, P_{z}$ are the generators of the symmetry. Since the Hamiltonian is degenerate, the components of $P$ need to be used for the theoretical description of the system: usually any two of them are added to $H_{\text {free }}$ to constitute a complete set of commuting observables (CSCO), $\left\{H_{\text {free }}, P_{x}, P_{y}\right\},\left\{H_{\text {free }}, P_{y}, P_{z}\right\}$ or $\left\{H_{\text {free }}, P_{x}, P_{z}\right\}$, that defines a basis of the Hilbert space. But this fact does not mean that those observables have to be considered definitevalued; in fact, this would be not possible since $P_{x}, P_{y}, P_{z}$ do not commute to each other. The MHI is completely consistent with this fact: according to its actualization rule, $H_{\text {free }}$ acquires a definite value, and also $P^{2}$ since it is proportional to $H_{\text {free }}$; nevertheless, $P_{x}, P_{y}, P_{z}$ are not definite-valued because the actualization of any of them would introduce an asymmetry not contained in the Hamiltonian: it would arbitrarily break the symmetry of the free particle. This result of the application of the MHI agrees with the empirical non-accessibility to the values of $P_{x}, P_{y}, P_{z}$ in the free particle. If we want to know those values, we must perform a measurement on the particle, in particular, a measurement that involves an interaction that breaks the symmetry of the original system by modifying its Hamiltonian. For instance, we can introduce a screen acting as a potential barrier that breaks the homogeneity of space. This means that, under measurement, the particle is no longer free: the symmetry breaking introduced by the interaction with the measuring system is what allows us to have empirical access to an observable that was a symmetry generator of the original free system.

In the case of the free particle with spin, the Hamiltonian is

$$
H=H_{\text {free }}+H_{\text {spin }}=\frac{P^{2}}{2 m}+k S^{2}
$$

According to the MHI, in this case the system is composite because it can be decomposed into two non-interacting subsystems: a free particle without spin, represented in $\mathcal{H}_{\text {free }}$ and with Hamiltonian $H_{\text {free }}=P^{2} / 2 m$, on which the rule applies as explained above, and a spin system, represented in $\mathcal{H}_{\text {spin }}$ and with Hamiltonian $H_{\text {spin }}=k S^{2}$, with $k=$ const. The spin subsystem is invariant under space-rotation: the generators of this symmetry are the three components $J_{x}, J_{y}, J_{z}$ of the total angular momentum $J$. But since in this case the orbital 
angular momentum $L$ is zero, the total angular momentum $J=L+S$ turns out to be simply $J=S$, and the three components $S_{x}, S_{y}, S_{z}$ of the spin $S$ are the generators of the spacerotation symmetry. As in the previous case, the three observables $S_{x}, S_{y}, S_{z}$ cannot simultaneously be definite-valued since they do not commute to each other. The MHI agrees with this fact: according to the actualization rule, in this case $H_{\text {spin }}$ acquires a definite value, and also $S^{2}$ since it is proportional to $H_{\text {spin }}\left(S^{2}\right.$ is the Casimir operator of the group generated by $S_{x}, S_{y}, S_{z}$ ); nevertheless, $S_{x}, S_{y}, S_{z}$ are not definite-valued since they are the generators of the space-rotation symmetry, and the actualization of any of them would break the symmetry of $H_{\text {spin }}$ in an arbitrary way. Again, this conclusion agrees with the fact that we have no empirical access to the spin components of the free particle with spin. If we want to know the value of those components, we have to perform a measurement on the system: we have to introduce a magnetic field $B$ of modulus $|B|$ in some direction, say $z$, which breaks the isotropy of space and, as a consequence, the original space-rotation symmetry. Under the action of $B$, the Hamiltonian $H_{\text {spin }}$ is not invariant under space-rotation anymore, because now it includes an interaction proportional to $|B| S_{z}$ that privileges a particular direction of space. In other words, we can have experimental access to the spin component $S_{z}$ only by means of a measurement that breaks the space-rotation symmetry of the original Hamiltonian and, therefore, makes the system no longer free. This is the usual way in which a spin component is measured in a Stern-Gerlach experiment.

The Hamiltonian of the hydrogen atom reads

$$
H_{\text {free }}=\frac{P_{e}^{2}}{2 m_{e}}+\frac{P_{p}^{2}}{2 m_{p}}+\frac{e^{2}}{\left|Q_{e}-Q_{p}\right|}
$$

where the subindexes $e$ and $p$ refer to the electron and to the proton respectively, and $e$ is the electric charge of the electron. When the spin of the electron is not considered, the atom is invariant under space-rotation, and the total angular momentum $J=L+S$ is simply $J=L$. Then, the three components $L_{x}, L_{y}, L_{z}$ of $L$ are the generators of the symmetry group. The possible states of the atom are labeled by the quantum numbers: the principal quantum number $n$, the orbital angular momentum quantum number $l$ and the magnetic quantum number $m_{l}$, which correspond to the eigenvalues of the observables $H, L^{2}$ and $L_{z}$ respectively. Since the Hamiltonian is degenerate due to its space-rotation invariance, the hydrogen atom is described in terms of the basis $\left\{\left|n, l, m_{l}\right\rangle\right\}$ defined by the CSCO $\left\{H, L^{2}, L_{z}\right\}$. Nevertheless, its space-rotation invariance makes the selection of $L_{z}$ a completely arbitrary decision: given that space is isotropic, we can choose $L_{x}$ or $L_{y}$ to obtain an equally legitimate description of the free atom. The arbitrariness in the selection of the $z$-direction is manifested 
in spectroscopy by the fact that the spectral lines of the free hydrogen atom give no experimental evidence about the values of $L_{z}$ : we have no empirical access to the number $m_{l}$ of the free atom. The MHI agrees with those experimental results since it does not assign a definite value to $L_{z}$ : the actualization of the value of $L_{z}$ would arbitrarily break the symmetry of the Hamiltonian of the free hydrogen atom.

Analogously to the measurement on a free particle with spin, in the case of the hydrogen atom a magnetic field $B$ along the $z$-axis breaks the isotropy of space and, as a consequence, the space-rotation symmetry of the atom's Hamiltonian. In this case, the symmetry breaking removes the energy degeneracy in $m_{l}$ : now $L_{z}$ is not arbitrarily chosen but selected by the direction of the magnetic field. But, in turn, this implies that the atom is no longer free: the Hamiltonian of the new system is approximately

$$
H=H_{\text {free }}+\frac{e}{2 m_{e} e} B L
$$

where $H_{\text {free }}$ is the Hamiltonian of the free atom. As a consequence, the original degeneracy of the $(2 l+1)$-fold multiplet of fixed $n$ and $l$ is now removed: the energy levels turn out to be displaced by an amount $\Delta \omega_{n l m_{l}}$, which is also function of $m_{l}:$ this is the manifestation of the so-called Zeeman effect. This means that the Hamiltonian, with eigenvalues $\omega_{n l m_{l}}$, is now non-degenerate: it constitutes by itself the CSCO $\{H\}$ that defines the preferred basis $\left\{\left|n, l, m_{l}\right\rangle\right\}$. According to the MHI actualization rule, in this case $H$ and all the observables commuting with $H$ are definite-valued: since this is the case for $L^{2}$ and $L_{z}$, in the physical conditions leading to the Zeeman effect both observables acquire definite values.

When the spectral lines of the hydrogen atom corresponding to $n>1$ are examined at a very high resolution, they are found to be closely spaced doublets: the energy levels of the atom are affected by the "coupling" between the electron spin $S$ and the orbital angular momentum $L$. Now the Hamiltonian of the system reads

$$
H=H_{\text {free }}+H_{\text {spin }}+H_{s-o}
$$

where $H_{\text {free }}$ is again the Hamiltonian of the free atom, $H_{\text {spin }}=k S^{2}$ is the Hamiltonian of the spin, and $H_{s-o}$ is the Hamiltonian representing the spin-orbit interaction. When the spin-orbit interaction is neglected ( $H_{s-o}=0$ ), the system is composite and can be described in terms of the basis $\left\{\left|n, l, m_{l}, s, m_{s}\right\rangle=\left|n, l, m_{l}\right\rangle \otimes\left|s, m_{s}\right\rangle\right\}$, where the $s(s+1) \hbar^{2}$ are the eigenvalues of $S^{2}$, and the $m_{s} \hbar$ are the eigenvalues of $S_{z}$. But when the spin-orbit interaction is taken into account, the observables $L_{z}$ and $S_{z}$ no longer commute with $H$ and, therefore, they are not constants of motion of the system: it is usually said that $m_{l}$ and $m_{s}$ are not good quantum 
numbers anymore. Nevertheless, the Hamiltonian is still invariant under space-rotation: the components $J_{x}, J_{y}, J_{z}$ of the total angular momentum $J$ are the generators of the symmetry group. In turn, $J=L+S$ is the sum of the orbital angular momentum $L$ and the spin angular momentum $S$, and $m_{j}=m_{l}+m_{s}$, where $m_{j}$ corresponds to the eigenvalue of $J_{z}$ . So, now $m_{j}$ is a good quantum number. Then, the basis $\left\{\left|n, l, j, s, m_{j}\right\rangle\right\}$ of the Hilbert space of the system is defined by the CSCO $\left\{H, L^{2}, J^{2}, S^{2}, J_{z}\right\}$. In this case, the spin-orbit coupling removes the original degeneracy of the eigenvalues $\omega_{n l}$ of the atom with no coupling; therefore, the MHI actualization rule selects $L^{2}, J^{2}$ and $S^{2}$ as definite-valued observables, because all of them commute with $H$ and have the same degeneracy in $m_{j}$ as $H$. But the space-rotation symmetry still present in the system leads to a degeneracy of $H$, manifested by the fact that the energy eigenvalues $\omega_{n l j s}$ do not depend on $m_{j}$. Then, according to the MHI, although in this case $m_{j}$ is a good quantum number, $J_{z}$ does not acquire a definite value, and this result agrees with the arbitrariness of the selection of the $z$-direction for $J_{z}$.

When a magnetic field is applied to the atom, the spectral lines split in different ways. The "normal" Zeeman effect, explained above, is observed in spin 0 states where, obviously, the spin-orbit coupling has no effect. In the states where the spin-orbit coupling is effective, the action of the magnetic field produces a further splitting of the energy levels known as "anomalous" Zeeman effect. Nevertheless, the explanation of the anomalous effect is the same as that of the normal effect: the action of the magnetic field along the $z$-axis breaks the space-rotation symmetry of the Hamiltonian by privileging the $z$-direction, and this leads to the removal of the original degeneracy of the Hamiltonian in the quantum number $m_{j}$ (instead of in the quantum number $m_{l}$ as in the normal effect). In this case, the MHI actualization rule prescribes that $J_{z}$ will be also definite-valued, in agreement with the experimental accessibility of $m_{j}$.

All the cases described above point to a feature of the quantum measurement that is not noticed in the usual, merely formal treatments of the process. In fact, in the von Neumann model, the observable $A$ to be measured on the system $S$ of interest is considered in formal terms and deprived of its physical content. Then, the interaction between $S$ and the measuring apparatus $M$ is endowed with the only role of introducing the correlation between $A$ and a pointer $P$. However, the physical situations just considered show that we have no empirical access to the observables that are generators of the symmetries of the system's Hamiltonian; and, in the context of measurement, $A$ may be one of those observables. This is precisely the case in the Stern-Gerlach experiment, where $S_{z}$ is a generator of the spacerotation symmetry of $H_{\text {spin }}=k S^{2}$; it is the interaction with the magnetic field $B=B_{z}$ that 
breaks the isotropy of space by privileging the $z$-direction and, as a consequence, breaks the space-rotation symmetry of $H_{\text {spin }}$.

This physical account of quantum measurement shows that, when the observable $A$ to be measured on the system $S$ is a generator of a symmetry of the Hamiltonian $H_{S}$ of $S$, the interaction with the apparatus $M$ not only must establish a correlation between $A$ and the pointer $P$, but also must break that symmetry. Therefore, from a physical viewpoint, measurement can be conceived as a process that breaks the symmetries of the system to be measured and, in this way, allows us to have access to an otherwise empirically inaccessible symmetry-generator observable. This suggests that the formal von Neumann model of quantum measurement should be complemented by a physical model in terms of which measurement is a symmetry breaking process that renders a symmetry generator of the system's Hamiltonian empirically accessible.

\section{6.- The modal-Hamiltonian account of optical isomerism}

In the light of the above account of quantum measurement, now Hund's paradox can be rephrased in MHI's language.

As explained in Section 3, the exact Hamiltonian $H$ of an enantiomer molecule is symmetric under spatial reflection: it commutes with the parity observable $P$ (see eq. (1)). Now, let us consider the observable chirality $C$, whose eigenstates are $|D\rangle$ and $|L\rangle$ : the eigenvalues $d$ and $l$ of $C$ represent the properties dextro-rotation and levo-rotation, respectively. The observable $C$ should be definite-valued for the molecule to be dextrorotatory (D) or levo-rotatory (L).

It is easy to see that $C$ does not commute with $H:[C, H] \neq 0$. As in the examples of the previous section, in this case the actualization of the observable $C$ would determine the chirality of the molecule in a completely arbitrary way: it would introduce in the molecule an asymmetry not contained in its Hamiltonian. As a consequence, from the MHI viewpoint, the observable chirality $C$ of the enantiomer molecule is not definite-valued, that is, it does not belong to the preferred context. In other words, strictly speaking the isolated molecule is not dextro-rotatory (D) or levo-rotatory (L): chirality is a property that has no definite value in it.

The fact that chirality does not have a definite value in the isolated molecule is compatible with experience: we have no experimental evidence of the handedness of an isolated molecule. In order to make chirality manifest as a definite-valued property, it is necessary to interact with the molecule. But the interaction leading to the expression of 
handedness must be such that it breaks the parity symmetry of the original Hamiltonian $H$. More precisely, the molecule must interact with another system $M$, which plays the role of the apparatus, in such a way that the Hamiltonian $H_{T}$ of the new composite system is no longer parity invariant. For instance, this happens when

$$
H_{T}=H+H_{M}
$$

where the Hamiltonian $H_{M}$ of the new system breaks the original parity invariance of $H$ : $\left[H_{M}, P\right] \neq 0 \rightarrow\left[H_{T}, P\right] \neq 0$. Additionally, $C$ must commute with the total Hamiltonian $H_{T}$ in order to obtain a stable reading of chirality. Under these conditions, according to the MHI the observable $C$ acquires a definite actual value: we measure dextro-rotation or levo-rotation. However, now the system is no longer the isolated molecule, but the molecule in interaction with the measuring system $M$.

This is precisely what happens when the molecule rotates the plane of polarization of plane-polarized light. In this case, a good candidate for $H_{M}$ is the Hamiltonian usually introduced in quantum chemistry to describe the interaction between molecules and polarized light (see Shao and Hänggi 1997), which is a function of the electric field $\bar{E}$ and the magnetic field $\bar{B}$ of the light. Therefore, the observable $C$ turns out to be a definite-valued observable of the new composite system. In other words, chirality is not an intrinsic property of the molecule, but of the system 'molecule plus light'. In a certain sense, this answer to Hund's paradox agrees with the view according to which the solution must be sought in the interaction of the molecule with its environment. However, our approach does not appeal to decoherence, but relies on an interpretation of quantum mechanics that explicitly accounts for measurement from the perspective of the symmetries of the system.

It is important to stress that, from the MHI viewpoint, symmetries are not conceived as generated by the spatial shapes of molecules: symmetries are internal features of the Hamiltonian of the whole closed system; they do not have to be conceived in terms of spatial configurations. According to the MHI, it is not correct to suppose that an enantiomer molecule has, before being observed, a definite handness depending on its shape, and that such a property is discovered when the molecule interacts with a system with the opposed handness. From the MHI perspective, isolated molecules have no definite chirality; chirality acquires a definite value when the molecule becomes a part of a composite system that is no longer parity symmetric.

The problem of isomerism is a particular case of what can be called, following Woolley and Sutcliffe (1977), the symmetry problem in chemistry: if the interactions embodied in the 
Hamiltonian of the molecule are Coulombic, the solutions of the Schrödinger equation have certain symmetries that cannot account for the asymmetries of the molecular structure. For instance, Coulombic molecules are spherically symmetrical; however, the asymmetry of polyatomic molecules is essential in the explanation of their chemical behavior. As Hendry stresses, according to quantum mechanics an isolated molecule in a general energy eigenstate may possess no directional property (Hendry 1998, p.131). The author considers the example of the hydrogen chloride molecule, which has an asymmetrical charge distribution that explains its acidic behavior and its boiling point; however, according to quantum mechanics, the expectation value of the dipole moment of a molecule in an arbitrary eigenstate of the full molecular Hamiltonian is always zero. On this basis, he concludes that "if the acidic behaviour of the hydrogen chloride molecule is conferred by its asymmetry, and the asymmetry is not conferred by the molecule's physical basis according to physical laws, then surely there is a prima facie argument that ontological reduction fails." (Hendry 2010, p. 186). The MHI casts new light on this problem, since it suggests that, although the isolated molecule lacks the necessary asymmetry, an adequate interaction may break the symmetry of the original Hamiltonian in the way needed to explain the asymmetric features that become manifest when the system is experimentally measured.

\section{7.- Conclusions}

In the context of the debates about the nature of molecular structure, in this paper we have focused on Hund's paradox, derived from the difficulty in giving a quantum explanation to optical isomerism. We have revised different attempts to solve the paradox, in particular, the widespread appeal to decoherence in recent literature; we have argued that none of these attempts is completely successful. On this basis, we have recalled the main features of the MHI in order to show how this interpretation offers a solution of Hund's paradox in exclusively quantum terms.

Since its first formulation in 2008, the MHI was developed in several articles, and was presented many times to varied audiences. It is interesting to notice the different reactions to the same proposal. Philosophers of physics usually insist on requiring the justification for the selection of the Hamiltonian as the key to determine the preferred context, by claiming that no basis of the Hilbert space has a privileged status. By contrast, philosophers of chemistry (and chemists) are always surprised by our insistence in justifying that selection, since in chemistry it is usual to work in the basis of the energy. In fact, the MHI is in resonance with molecular chemistry, in the context of which molecules are almost always described in their stationary 
states, that is, in eigenstates of the Hamiltonian, and a great deal of relevant knowledge is obtained from studying the symmetries of the Hamiltonian. The MHI account to optical isomerism fits in the framework of this resonance.

\section{Acknowledgements:}

We want to acknowledge Marta Beatriz Ferraro, for her kind advice about the scientific practice related with optical isomerism. This article was made possible through the support of Grant 57919 from the John Templeton Foundation and Grant PICT-2014-2812 from the National Agency of Scientific and Technological Promotion of Argentina.

\section{References}

Adler, S. L. (1995). Quaternionic Quantum Mechanics and Quantum Fields. New York: Oxford University Press.

Adler, S. (2003). "Why decoherence has not solved the measurement problem: A response to P. W. Anderson.” Studies in History and Philosophy of Modern Physics, 34: 135-142.

Albert, D. and Loewer, B. (1990). "Wanted dead or alive: two attempts to solve Schrödinger's paradox." Pp. 277-285, in Proceedings of the PSA 1990, Vol. 1, A. Fine, M. Forbes and L. Wessels (eds.). East Lansing, Michigan: Philosophy of Science Association.

Albert, D. and Loewer, B. (1991). "Some alleged solutions to the measurement problem." Synthese, 88: 87-98.

Albert, D. and Loewer, B. (1993). "Non-ideal measurements." Foundations of Physics Letters, 6: 297-305.

Amann, A. (1992). "Must a molecule have a shape?" South African Journal of Chemistry, 45: 29-38.

Ardenghi, J. S., Castagnino, M. and Lombardi, O. (2009). "Quantum mechanics: modal interpretation and Galilean transformations." Foundations of Physics, 39: 1023-1045.

Ardenghi, J. S. and Lombardi, O. (2011). "The Modal-Hamiltonian Interpretation of quantum mechanics as a kind of "atomic" interpretation." Physics Research International, 2011: 379604. 
Ardenghi, J. S., Lombardi, O. and Narvaja, M. (2013). "Modal interpretations and consecutive measurements." Pp. 207-217, in EPSA 2011: Perspectives and Foundational Problems in Philosophy of Science, V. Karakostas and D. Dieks (eds.). Dordrecht: Springer.

Bacciagaluppi, G. (1995). "A Kochen-Specker theorem in the modal interpretation of quantum mechanics.” International Journal of Theoretical Physics, 34: 1205-1216.

Bacciagaluppi, G. (2012). "The role of decoherence in quantum mechanics.” In The Stanford Encyclopedia of Philosophy (Winter 2012 Edition), E. N. Zalta (ed.). URL = <http://plato.stanford.edu/ archives/ win2012/entries/qm-decoherence/>.

Bacciagaluppi, G. and Dickson, M. (1999). "Dynamics for modal interpretations." Foundations of Physics, 29: 1165-1201.

Bacciagaluppi, G. and Hemmo, M. (1996). "Modal interpretations, decoherence and measurements." Studies in History and Philosophy of Modern Physics, 27: 239-277.

Bene, G. and Dieks, D. (2002). “A perspectival version of the modal interpretation of quantum mechanics and the origin of macroscopic behavior." Foundations of Physics, 32: $645-671$.

Berlin, Y. A., Burin, A. L. and Goldanskii, V. V. (1996). "The Hund paradox and stabilization of molecular chiral states." Zeitschrift für Physik D, 37: 333-339.

Bishop, R. (2005). "Patching physics and chemistry together." Philosophy of Science, 72: 716-722.

Bishop, R. and Atmanspacher, H. (2006). "Contextual emergence in the description of properties." Foundations of Physics, 36: 1753-1777.

Bub, J. (1997). Interpreting the Quantum World. Cambridge: Cambridge University Press.

Castagnino, M. and Lombardi, O. (2008). "The role of the Hamiltonian in the interpretation of quantum mechanics.” Journal of Physics. Conferences Series, 28: 012014.

Chang, H. (2015). "Reductionism and the relation between chemistry and physics." Pp. 193210, in Relocating the History of Science: Essays in Honor of Kostas Gavroglu, T. Arabatzis, J. Renn and A. Simoes (eds.). New York: Springer.

Cohen-Tannoudji, C., Diu, B. and Lalöe, F. (1977). Quantum Mechanics. New York: John Wiley \& Sons. 
da Costa, N. and Lombardi, O. (2014). "Quantum mechanics: ontology without individuals." Foundations of Physics, 44: 1246-1257.

da Costa, N., Lombardi, O. and Lastiri, M. (2013). “A modal ontology of properties for quantum mechanics.” Synthese, 190: 3671-3693.

d'Espagnat, B. (1966). “An elementary note about mixtures.” Pp. 185-191, in Preludes in Theoretical Physics, A. De-Shalit, H. Feshbach and L. van Hove (eds.). Amsterdam: North-Holland.

d'Espagnat, B. (1976). Conceptual Foundations of Quantum Mechanics. Reading MA: Benjamin.

Dieks, D. (1988). "The formalism of quantum theory: an objective description of reality?" Annalen der Physik, 7: 174-190.

Dieks, D. (1989). "Quantum mechanics without the projection postulate and its realistic interpretation." Foundations of Physics, 38: 1397-1423.

Dirac, P. A. M. (1929). "Quantum mechanics of many-electron systems.” Proceedings of the Royal Society of London A, 123: 714-33.

Elby, A. (1993). "Why 'modal' interpretations of quantum mechanics don't solve the measurement problem." Foundations of Physics Letters, 6: 5-19.

Fock, F. (1935). “Zur Theorie des Wasserstoff Atoms.” Zeitschrift für Physik, 98: 145-154.

Fortin, S. and Lombardi, O. (2014). "Partial traces in decoherence and in interpretation: What do reduced states refer to?" Foundations of Physics, 44: 426-446.

Fortin, S., Lombardi, O. and Martínez González, J. C. (2016). "Isomerism and decoherence.” Foundations of Chemistry, on line first.

Harshman, N. L. and Wickramasekara, S. (2007a). "Galilean and dynamical invariance of entanglement in particle scattering." Physical Review Letters, 98: 080406.

Harshman, N. L. and Wickramasekara, S. (2007b). "Tensor product structures, entanglement, and particle scattering." Open Systems and Information Dynamics, 14: 341-351.

Harris, R. A. and Stodolsky, L. (1981). "Time dependence of optical activity.” The Journal of Chemical Physics, 74: 2145-2155.

Hendry, R. F. (1998). "Models and approximations in quantum chemistry." Pp. 123-142, in N. Shanks (ed.), Idealization in Contemporary Physics. Amsterdam-Atlanta: Rodopi. 
Hendry, R. F. (2004). "The physicists, the chemists, and the pragmatics of explanation." Philosophy of Science, 71: 1048-59.

Hendry, R. F. (2008). "Two conceptions of the chemical bond." Philosophy of Science, 75: 909-920.

Hendry, R. F. (2010). “Ontological reduction and molecular structure.” Studies in History and Philosophy of Modern Physics, 41: 183-191.

Hettema, H. (2012). Reducing Chemistry to Physics. Limits, Models, Consecuences. Groningen: University of Groningen.

Hund. F. (1927). “Zur Deutung der Molekelspektren. III.” Zeitschrift für Physik, 43: 805-826.

Joos, E. (1996). "Decoherence through interaction with the environment." Pp. 41-180, in Decoherence and the Appearance of a Classical World in Quantum Theory, E. Joos, H. D. Zeh, C. Kiefer, D. Giulini, J. Kupsch and I-O. Stamatescu (eds.). Heidelberg: Springer-Verlag.

Kochen, S. (1985). “A new interpretation of quantum mechanics.” Pp. 151-169, in Symposium on the Foundations of Modern Physics 1985, P. Mittelstaedt and P. Lahti (eds.). Singapore: World Scientific.

Kochen, S. and Specker, E. (1967). "The problem of hidden variables in quantum mechanics." Journal of Mathematics and Mechanics, 17: 59-87.

Lombardi, O. (2010). "The central role of the Hamiltonian in quantum mechanics: decoherence and interpretation.” Manuscrito, 33: 307-349.

Lombardi, O. and Castagnino, M. (2008). "A modal-Hamiltonian interpretation of quantum mechanics." Studies in History and Philosophy of Modern Physics, 39: 380-443.

Lombardi, O. and Castagnino, M. (2010). "Matters are not so clear on the physical side." Foundations of Chemistry, 12: 159-166.

Lombardi, O., Castagnino, M. and Ardenghi, J. S. (2010). "The modal-Hamiltonian interpretation and the Galilean covariance of quantum mechanics." Studies in History and Philosophy of Modern Physics, 41: 93-103.

Lombardi, O. and Dieks, D. (2014). "Modal interpretations of quantum mechanics." In The Stanford Encyclopedia of Philosophy (Spring 2014 Edition), E. N. Zalta (ed.). URL = <http://plato.stanford.edu/archives/spr2014/entries/qm-modal/>. 
Lombardi, O. and Dieks, D. (2016). "Particles in a quantum ontology of properties." Pp. 123143, in Metaphysics in Contemporary Physics, T. Bigaj and C. Wüthrich (eds.). Leiden: Brill.

Lombardi, O., Fortin, S., Castagnino, M. and Ardenghi, J. S. (2012). "Compatibility between environment-induced decoherence and the modal-Hamiltonian interpretation of quantum mechanics." Philosophy of Science, 78: 1024-1036.

Lombardi, O., Fortin, S. and López, C. (2015). "Measurement, interpretation and information." Entropy, 17: 7310-7330.

Masillo, F., Scolarici, G. and Sozzo, S. (2009). "Proper versus improper mixtures: towards a quaternionic quantum mechanics.” Theoretical and Mathematical Physics, 160: 10061013.

Primas, H. (1983). Chemistry, Quantum Mechanics and Reductionism. Berlin: Springer.

Primas, H. (1994). "Hierarchic quantum descriptions and their associated ontologies." Pp. 210-220, in Symposium on the Foundations of Modern Physics 1994, K. V. Laurikainen, C. Montonen and K. Sunnarborg (eds.). Gif-sur-Yvette: Editions Frontières.

Primas, H. (1998). "Emergence in exact natural sciences." Acta Polytechnica Scandinavica, 91: 83-98.

Ruetsche, L. (1995). "Measurement error and the Albert-Loewer problem." Foundations of Physics Letters, 8: 327-344.

Scerri, E. R. (2011). “Editorial 37.” Foundations of Chemistry, 13: 1-7.

Scerri, E. R. (2012). "Top-down causation regarding the chemistry-physics interface: a sceptical view." Interface Focus, 2: 20-25.

Scerri, E. R. (2013). "Philosophy of chemistry: where has it been and where is it going." Pp. 208-225, in J.-P. Llored (ed.), The Philosophy of Chemistry: Practices, Methodologies, and Concepts. Newcastle: Cambridge Scholars Publishing.

Schlosshauer, M. (2007). Decoherence and the Quantum-to-Classical Transition. Berlin: Springer.

Shao, J. and Hänggi, P. (1997). "Control of molecular chirality.” The Journal of Chemical Physics, 107: 9935-9941. 
Sutcliffe, B. T. and Woolley, R. G. (2011). “A comment on Editorial 37.” Foundations of Chemistry, 13: 93-95.

Sutcliffe, B. T. and Woolley, R. G. (2012). “Atoms and molecules in classical chemistry and quantum mechanics." Pp. 387-426, in Handbook of Philosophy of Science. Vol. 6, Philosophy of Chemistry, R. F. Hendry and A. Woody (eds). Oxford: Elsevier.

Tinkham, M. (1964). Group Theory and Quantum Mechanics. New York: McGraw-Hill.

Trost, J. and Hornberger, K. (2009). "Hund's paradox and the collisional stabilization of chiral molecules." Physical Review Letters, 103: 023202.

Tung, W. K. (1985). Group Theory in Physics. Singapore: World Scientific.

Van Fraassen, B. C. (1972). “A formal approach to the philosophy of science.” Pp. 303-366, in Paradigms and Paradoxes: The Philosophical Challenge of the Quantum Domain, R. Colodny (ed.). Pittsburgh: University of Pittsburgh Press.

Van Fraassen, B. C. (1974). “The Einstein-Podolsky-Rosen paradox.” Synthese, 29: 291-309.

Vermaas, P. (1997). "A no-go theorem for joint property ascriptions in modal interpretations of quantum mechanics.” Physical Review Letters, 78: 2033-2037.

Vermaas, P. and Dieks, D. (1995). "The modal interpretation of quantum mechanics and its generalization to density operators." Foundations of Physics, 25: 145-158.

Weyl, H. (1950). The Theory of Groups and Quantum Mechanics. New York: Dover (first edition in German, 1928).

Woolley, R. G. (1978). “Must a molecule have a shape?" Journal of the American Chemical Society, 100: 1073-1078.

Woolley, R. G. (1982). "Natural optical activity and the molecular hypothesis." Structure and Bonding, 52: 1-35.

Zurek, W. H. (1981) "Pointer basis of quantum apparatus: into what mixture does the wave packet collapse?" Physical Review D, 24: 1516-1525.

Zurek, W. H. (1991). "Decoherence and the transition from quantum to classical." Physics Today, 44: 36-44.

Zurek, W. H. (2003). "Decoherence, einselection, and the quantum origins of the classical." Reviews of Modern Physics, 75: 715-776. 